\title{
Determination of critical stresses during prestressing along the entire length of a circular-cylindrical shell
}

\author{
Takhir Chapaev ${ }^{1, *}$, and Nurilla Noraliev $^{2}$ \\ ${ }^{1}$ Kabardino-Balkarian State Agricultural University named after V. M. Kokov, Lenin Avenue, 1V, \\ 360030 Nalchik, Russia \\ ${ }^{2}$ Tashkent State Agrarian University, Universitetskaya, 2, 100140 Kibray district, Tashkent region, \\ Republic of Uzbekistan
}

\begin{abstract}
In the Russian Federation, there is an urgent issue of grain storage and processing to be resolved through the reconstruction of old and construction of new storage facilities. Currently, the most common construction is represented by steel granaries, erected by rolling using prestressing in shells. The relevance of the problem of stability of the wall of a granary, taking into account the main factors, affecting the strength, requires further theoretical and experimental study. One of the most frequently used in solving stability problems is the energy criterion in the form of Ritz-Timoshenko, which makes it possible to determine critical stresses in the shells. In this case, the problem solution becomes simpler, it is easier to consider the effect of such factors as initial perfection, friction forces between the shell and the winding, as well as other features of the stability problem of a prestressed shell. This article analyses the granary wall stability against lateral external pressure of prestressed winding or bandages. For large tanks, prestressing is generated along the entire length of the shell, and in vertical cylindrical granaries, prestressing is generated on a part of the length (height) of the shell.
\end{abstract}

\section{Introduction}

The actual ratios of tank diameters and granaries and their heights show that their bodies are shells of medium length.

The stability of cylindrical shells of medium length under the action of lateral uniform external pressure has been devoted to many works $[1,2,3,4,5]$. The analysis of these works shows that the effect of shell end grounding on its stability depends on the shell relative length.

Figure 1 shows the numerical analysis results of the effect of various conditions of the shell fastening on its stability from lateral uniform pressure [5]. The following designations are used here:

\footnotetext{
*Corresponding author: 7227229@mail.ru
} 


$$
Z_{0}=\frac{L \sqrt[4]{1-\mu^{2}}}{\sqrt{R t_{1}}} ; \varsigma_{0}=\frac{q_{\kappa p}}{q_{k p}^{*}}
$$

whe $L \quad-$ shell length;

re:

$$
\begin{array}{ll}
t_{1} & - \text { shell thickness; } \\
R & - \text { shell radius; } \\
\mu & - \text { Poisson's ratio; } \\
q_{k p}^{*} & - \text { determined by the formula of P. F. Papkovich [1]. }
\end{array}
$$

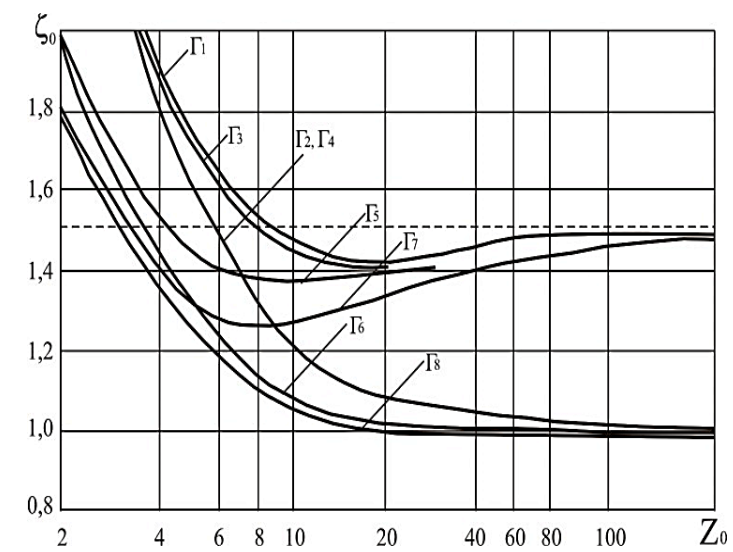

Fig. 1. Effect of boundary conditions on the cylindrical shell stability against lateral external pressure.

The shown curves correspond to the following variants of the boundary conditions at both shell ends:

$$
\begin{aligned}
& \Gamma_{1} \quad w=0, \quad \frac{\partial w}{\partial x}=0, \quad U=0, \quad V=0 \\
& \Gamma_{2} \quad w=0, \quad \frac{\partial w}{\partial x}=0, \quad T_{x}=0, \quad V=0 ; \\
& \Gamma_{3} \quad w=0, \quad \frac{\partial w}{\partial x}=0, \quad U=0, \quad S_{x y}=0 ; \\
& \Gamma_{4} \quad w=0, \quad \frac{\partial w}{\partial x}=0, \quad T_{x}=0, \quad S_{x y}=0 ;, \\
& \Gamma_{5} \quad w=0, \quad \frac{\partial w}{\partial x}=0, \quad U=0, \quad V=0 ; \\
& \Gamma_{6} \quad w=0, \quad M_{x}=0, \quad T_{x}=0, \quad V=0 ; \\
& \Gamma_{7} \quad w=0, \quad M_{x}=0, \quad U=0, \quad S_{x y}=0 ; \\
& \Gamma_{8} \quad w=0, \quad M_{x}=0, \quad T_{x}=0, \quad S_{x y}=0 ;
\end{aligned}
$$

whe $U$ and $V-$ respectively, longitudinal and circumferential displacements of points re: of the shell middle surface;

$T_{x} \quad$ and - longitudinal and shear forces in the shell. $S_{x y}$

Parameter $z_{0}$ for vertical cylindrical tanks and granaries with a volume of 0.5 to 100 thous. $\mathrm{m}^{3}$ is in the range from 20 to 80 . The actual boundary conditions for fixing the body wall occupy an intermediate position between 2 and 8 variants of the boundary conditions 
given above. Thus, judging by the curves corresponding to variants 2 and 8 of the boundary conditions, we can say that for vertical cylindrical tanks and granaries with a volume of 5 to 100 thous. $\mathrm{m}^{3}$, the presence of elastic grounding of the wall at the bottom practically does not affect the stability against lateral external pressure of the vacuum type. In other words, in calculating the stability of vertical cylindrical tanks or granaries of the indicated volume, it can be taken as a margin of stability that the body wall is hinged at the ends.

In the case of a spiral-wound silo, the stability problem of a separate shell is solved, $L \approx 40 \mathrm{~cm}$ long, parameter $z_{0}$ has a value $z_{0}=2 \div 3$.

According to Fig. 1, the presence of wall clamping in the folds should increase its resistance to lateral pressure. However, in this case, we also assume that the shell is hinged at the ends as a margin of stability.

This article analyses the tank shell wall stability against lateral external pressure of a prestressed winding or bandages. For large tanks, prestressing is generated along the entire length of the shell, and in vertical cylindrical granaries, prestressing is generated on a part of the length (height) of the shell.

The shell stability is lost in one of the sections with the most significant initial dents. In the remaining sections, as the compression force increased, there was a slight increase in dents, however, with a loss of stability in one of shell sections, deflections stop growing, since forces in the winding or bandages decrease. Thus, for the limiting state of stability loss, we take the moment of the formation of a dent in one of shell sections.

\section{Materials and Methods}

The problem was solved using the Ritz-Timoshenko energy method. The energy balance equation for the prestressed shell is written in the form:

$$
\Delta U=U_{o \sigma m}+U_{m p}+U_{c}+U_{H}+\Pi-U_{0}=0,
$$

whe $U_{\text {обм }}$ - potential energy of winding deformation during shell buckling;

re:

$U_{m p}-$ potential energy of friction forces arising between the shell and the winding during their mutual displacement at the moment of buckling;

$U_{c}-$ potential deformation energy of the shell middle surface;

$U_{H}-$ potential energy of shell bending;

$\Pi \quad-$ potential of winding pressure forces on the shell;

$U_{0}-$ potential energy of a prestressed shell before stability loss.

The critical force in the winding $N_{k p}$ is defined as the minimum tension force in the winding $N_{0}$, when the potential energy of the shell-winding system before buckling will be equal to the potential energy of the system in a deformed state after buckling.

To determine the parameters included in equation (3), it is necessary to set the shell deflection line.

To approximate the shell deflections, an expression is adopted that sufficiently corresponds to the real buckling shape shown in Figure 2:

$$
w=f \cos \eta \theta \sin \frac{\pi x}{L},
$$

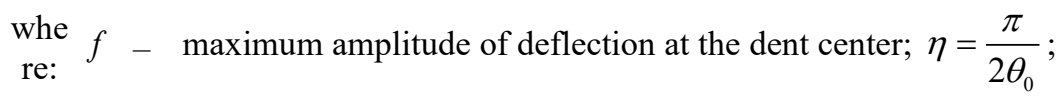


$\theta_{0}-$ the central angle defining the zone of winding separation from the shell during buckling.
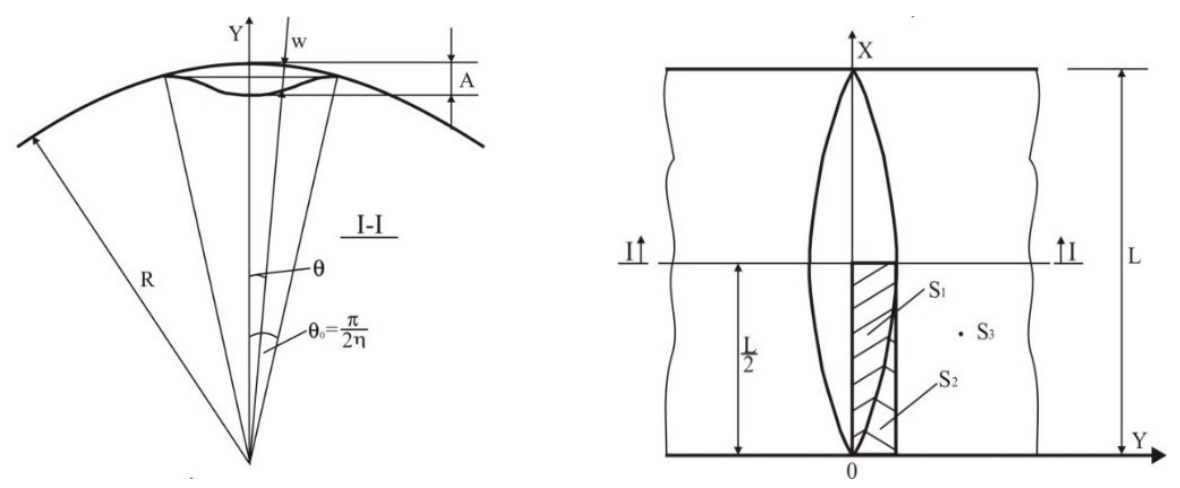

Fig. 2. Scheme of shell buckling along the entire winding length.

Formula (4) is valid for $0<\theta \leq \theta_{0}$.

With the indicated indentation scheme, the value $f$ can be determined through shell radius $R$ and angle $\theta_{0}$ by the formula:

$$
f=2,5 R \sin \theta_{0} t q \theta_{0},
$$

where coefficient 2.5 is taken on the basis of experimentally obtained shape of the dent formed during stability loss and the analysis of the relationship between the value of shell deflections and delamination of the winding from the shell.

Thus, taking into account formula (5), the variable deflection parameter is angle $\theta_{0}$. In other words, we assume that the shape of the initial wave formation is in "resonance" with the wave formation of the shell in the process of deformation, and that the only preset parameter is the maximum value of the initial dents $A_{0}$. This assumption somewhat enhances the effect of the initial inaccuracies, but it greatly simplifies the mathematical calculations. It is mentioned in works of L. G. Donell [5] and A. S. Volmir [6].

In real forms of initial dents, in contrast to those taken by formula (5), there is a buckling for the outer contour. The pressure from the prestressed winding in these areas with greater curvature is higher than in the areas with less curvature. Therefore, the real working conditions of the wall in the zone with stability loss can be considered similar to those assumed in this solution. That is, we assume that the shape of the initial indentations corresponds to formula (5) and that the pressure from the winding or bands is the same in all areas of their contact with the shell.

As a result of shell buckling and dent formation, the winding circumference is less than that of the shell. As, the force in the winding is reduced. The relative winding deformation $\xi_{1}$ is determined from the condition of compatibility of shell and winding deformation:

$$
\xi_{1}=1-\frac{S_{1}+S_{2}+S_{3}}{2 \pi R L}
$$

whe $S_{1}, S_{2}, S_{3}-$ sections of the reduced winding surface area re: after shell buckling (see Fig. 2), determined by the formulas:

$$
S_{1}=2 R \int_{0}^{L} \sin \theta_{0} \sin \frac{\pi x}{L} d x=\frac{4 R L}{\pi} \sin \frac{\pi}{2 \eta} ;
$$




$$
\begin{gathered}
S_{2}=4 R \int_{0}^{L} \frac{\pi}{2 \eta}\left(\frac{1}{2}-\frac{1}{\pi}\right) d x=\frac{2 \pi R L}{\eta}\left(0,5-\frac{1}{\pi}\right) \\
S_{3}=2 R \int_{0}^{L}\left(\pi-\theta_{0}\right) d x=2 \pi R L\left(1-\frac{1}{2 \eta}\right)
\end{gathered}
$$

After substituting them into formula (6), we have:

$$
\xi_{1}=\frac{1}{\eta \pi}-\frac{2}{\pi^{2}} \sin \frac{\pi}{2 \eta}
$$

Taking into account the initial dent with an amplitude in the middle equal to $f_{0}$, the relative winding deformation with the shell stability loss will be less by an amount $\xi_{0}$ that can be determined as

$$
\xi_{0}=\frac{1}{\eta_{0} \pi}-\frac{2}{\pi^{2}} \sin \frac{\pi}{2 \eta_{0}}
$$

where: $\eta_{0}=\pi \sqrt{0,625 \frac{R}{f_{0}}}$ characterizes the initial dent width.

Then the value of the winding relative deformation, taking into account formulas (7) and (8), will be determined by the formula:

$$
\xi=\xi_{1}-\xi_{0}=\frac{1}{\pi \eta}-\frac{1}{\pi \eta_{0}}-\frac{2}{\pi^{2}}\left(\sin \frac{\pi}{2 \eta}-\sin \frac{\pi}{2 \eta_{0}}\right) .
$$

It makes it possible to proceed to the determination of the potential energy of deformation of the prestressed winding as a result of its length reduction during shell buckling. After loss of stability, the force in the winding will decrease by an amount $E_{2} t_{2} \xi=B_{2} \xi$.

\section{Results and Discussion}

In the presence of significant dents $\left(2 \div 5 t_{1}\right)$ and with a decreasing load, the process of stability loss is not so dynamic and is closer to the inertialless process. In this case, the behavior of the system is determined by the diagram of equilibrium states, and at any stage of deformation its resistance is equal to the load. The foregoing allows us to assume that the deformation of the winding occurs at an average value between the force before buckling and the force after buckling, i.e. with force. $N_{0} N_{0}-B_{2} \xi N_{0}-0,5 B_{2} \xi$ Then the potential energy of winding deformation can be determined by the formula:

$$
U_{\text {обм }}=\frac{t_{2}}{E_{2}} \int_{0}^{L} R \int_{0}^{\pi} \frac{\left(N_{0}-0,5 B_{2} \xi\right)^{2}}{t_{2}^{2}} d \theta d x=\frac{\pi R L}{B_{2}}\left(N_{0}-0,5 B_{2} \xi\right)^{2} .
$$

The energy of friction forces in this case is determined by the formula:

$$
U_{m p}=2 \int_{0}^{L} R \int_{\frac{\theta_{0}}{2}}^{\pi} f_{m p} \frac{N_{0}}{R} \xi(\pi-\theta) R d \theta d x=N_{0} f_{m p} \xi R L\left(\pi-\frac{\pi}{4 \eta}\right)^{2} .
$$

The resulting formula makes it possible to approximately consider the effect of friction forces on the shell stability against the action of prestressing, since it does not take into account initial dents along the shell perimeter, they reduce shell contact zone with the 
winding, but also does not take into account the increase in radial pressure forces where the initial dents have a shape with slight bulging behind the outer contour, resulting in an increase in the shell curvature. Besides, a certain dynamism of the winding shift relative to the shell is not considered. Taking into account all these factors is a rather difficult task, difficult to accomplish within the framework of this article.

Shell buckling, as a rule, is accompanied by not only bending stresses, but also additional stresses in the middle surface. Part of the external load potential upon stability loss of the shell is spent on increasing the bending energy, and the other part - on changing the deformation energy of the middle surface. The ratio depends on the shell size and configuration taken on buckling. For medium length shells, the deformation energy of the middle surface becomes commensurate with shell bending energy. To determine the deformation energy, it is necessary to determine the stress distribution function in the middle surface $\Phi$.

Since the shell buckling is accompanied by the dent formation with a large deflection amplitude, the problem should be solved in a geometrically nonlinear setting. To determine the stress distribution function in the middle surface, we will solve the fourth-order nonlinear differential equation of compatibility of deformations, used in the theory of flexible shells.

As there are initial decays in real shells, we use the equation given in $[7,8,9]$ :

$$
\frac{1}{E_{1}} \nabla^{4} \Phi=-\frac{1}{2}\left[L_{(w, w)}-L_{\left(w_{0}, w_{0}\right)}\right]-\frac{1}{R} \frac{\partial^{2}\left(w-w_{0}\right)}{\partial x^{2}},
$$

where operators $L_{(w, w)}$ and $L_{\left(w_{0}, w_{0}\right)}$, being nonlinear terms of this differential equation, are determined by the formulas [10]:

$$
\begin{aligned}
L_{(w, w)} & =2\left[\frac{\partial^{2} w}{\partial x^{2}} \frac{\partial^{2} w}{R^{2} \partial \theta^{2}}-\left(\frac{\partial^{2} w}{\partial x R \partial \theta}\right)^{2}\right], \\
L_{\left(w_{0}, w_{0}\right)} & =2\left[\frac{\partial^{2} w_{0}}{\partial x^{2}} \frac{\partial^{2} w_{0}}{R^{2} \partial \theta^{2}}-\left(\frac{\partial^{2} w_{0}}{\partial x R \partial \theta}\right)^{2}\right] .
\end{aligned}
$$

To solve the differential equation (11), we use the following boundary conditions at shell ends (with hinged fastening): at $x=0$ and $x=L, w=0$ and $\frac{\partial^{2} w}{\partial x^{2}}=0$.

After integrating (11), taking into account formulas (12) and (13), we obtain the following expression for the stress distribution function in the shell middle surface $\Phi$ :

$$
\Phi=E_{1}\left(f^{2}-f_{0}^{2}\right) \beta \frac{\eta^{2}}{2 R^{2}}\left(\cos \frac{2 \pi x}{L}-\cos 2 \eta \theta\right)+E_{1} \frac{f-f_{0}}{R} \beta \cos \eta \theta \sin \frac{\pi x}{L}-\frac{N_{0}}{t_{1}} \frac{x^{2}}{2},
$$

where: $\beta=\frac{\left(\frac{\pi}{L}\right)^{2}}{\left(\frac{\pi^{2}}{L^{2}}+\frac{\eta^{2}}{R^{2}}\right)^{2}}$.

Formula (14) is valid for $0 \leq \theta \leq \theta_{0}$.

The potential energy of deformation of the shell middle surface is determined taking into account a similar formula [4]: 


$$
U_{c}=\frac{t_{1}}{E_{1}} R \int_{0}^{L} \int_{0}^{L}\left[\left(\nabla^{2} \Phi\right)^{2}-(1+\mu) L_{(\Phi, \Phi)}\right] d x d \theta+\frac{t_{1}}{E_{1}} \int_{0}^{L} R \int_{\theta_{0}}^{\pi} \frac{N_{0}^{2}}{t_{1}^{2}} d x \theta,
$$

where: $L_{(\Phi, \Phi)}=2\left[\frac{\partial^{2} \Phi}{\partial x^{2}} \frac{\partial^{2} \Phi}{R^{2} \partial \theta^{2}}-\left(\frac{\partial^{2} \Phi}{\partial x R \partial \theta}\right)^{2}\right]$.

As a result of integration and some transformations, we get:

$$
U_{c}=B_{1} C_{1}^{\prime}+\frac{N_{0}^{2} \pi R L}{B_{1}},
$$

where $B_{1}=E_{1} t_{1}, \beta_{1}=\frac{\pi^{2}}{L^{2}}+\frac{\eta^{2}}{R^{2}}, C_{1}^{\prime}=\left(f^{2}-f_{0}^{2}\right)^{2} \beta^{2}\left(\frac{\eta^{3} \pi^{5}}{R^{3} L^{3}}+\frac{\pi \eta^{3} L}{R^{3}}\right)+\left(f-f_{0}\right)^{2} \frac{\beta^{2} \beta_{1}^{2}}{8 \eta R} \pi L$.

The potential bending energy of the shell is determined taking into account the formula (12.95) [4]. However, in our problem, it is also necessary to take into account the bending stiffness of the winding in the annular direction. An approximate expression for the potential bending energy, taking into account the initial deflection, will have the form:

$$
\begin{gathered}
U_{H}=D R \int_{0}^{L} \int_{0}^{\theta_{0}}\left[\left(\nabla^{2} w-w_{0}\right)^{2}-(1-\mu) L_{\left(w-w_{0}, w-w_{0}\right)}\right] \partial \theta d x= \\
=\frac{\pi R L\left(f-f_{0}\right)}{8 \eta}\left[\frac{\pi^{4}}{L^{4}} D_{1}+2\left(\frac{\pi \eta}{L R}\right)^{2} D_{1}+\frac{\eta^{4}}{R^{4}}\left(E_{2} I_{2} \frac{1}{2}+D_{1}\right)\right]
\end{gathered}
$$

whe $D-$ a parameter that takes into account the stiffness of the shell for bending in the re: annular and longitudinal directions, as well as the winding stiffness for bending in the annular direction;

$$
\begin{aligned}
& D_{1}=\frac{E_{1} t_{1}^{3}}{12\left(1-\mu^{2}\right)}-\text { cylindrical shell stiffness; } \\
& E_{2} I_{2}=\frac{E_{2} t_{2}^{3}}{12}-\text { reduced bending stiffness of the winding. }
\end{aligned}
$$

It should be noted that formula (17) takes into account possible mutual slippage between the winding and the shell, therefore, the flexural stiffness for the shell and the winding is determined separately as for two unconnected elements.

When the shell bulges in the dent zone, the radial pressure of the winding decreases, depending on the change in the shell curvature, to $N_{0} / R$ zero. The larger the reduced winding thickness, the faster the tension force decreases, and, consequently, the radial pressure on the shell in this zone.

The potential of the radial pressure forces of the shell winding can be determined by analogy with the formula (13.91) [4]:

$$
\Pi=-2 R \int_{0}^{L} \int_{0}^{\theta_{0}} \frac{w}{2,5} \frac{1}{2} P d \theta d x=-\frac{N_{0}-B_{2} \xi}{2,5} \int_{0}^{L} \int_{0}^{\theta_{0}} \cos \eta \theta \sin \frac{\pi x}{L} d \theta d x=-\left(N_{0}-B_{2} \xi\right) C_{2}^{\prime},
$$

where: $P=\frac{N_{0}-B_{2} \xi}{R}-$ radial pressure of the winding on the shell after buckling;

$$
C_{2}^{\prime}=\frac{1}{1,25 \pi \eta}\left(f-f_{0}\right) L .
$$

It should be noted that the pressure $P$ can be considered equal to zero when, in the section where the shell buckling occurs, its curvature becomes zero, that is, when 
(according to the diagram in Fig. 2 and formula (5)) the shell deflection becomes equal to $w / 2,5$. Assuming that the winding pressure on the shell during buckling of the shell varies from $P$ to zero according to a linear law, the average value of the pressure performing the work can be taken as $0,5 P$. Accordingly, in formula (18), the deflection $w$ is divided by a factor of 2.5 , and the pressure by a factor of 2 .

The potential system energy before stability loss is determined by the expression:

$$
U_{0}=\frac{t_{1}}{E_{1}} R \int_{0}^{L} \int_{0}^{\pi} \frac{N_{0}^{2}}{t_{1}^{2}} d \theta d x+\frac{t_{2}}{E_{2}} \int_{0}^{L} R \int_{0}^{\pi} \frac{N_{0}^{2}}{t_{2}^{2}} d \theta d x=\pi R L N_{0}^{2}\left(\frac{1}{B_{1}}+\frac{1}{B_{2}}\right) .
$$

Substituting (10), (11), (16), (17), (18) and (19) into the energy balance equation (3), we solve it with respect to the winding tension force $N_{0}$ [10]:

$$
N_{0}=\frac{U_{H}+B_{1} C_{1}^{\prime}+B_{2} \xi\left(0 / 25 \pi R L \xi+C_{2}^{\prime}\right)}{\pi R L \xi+C_{2}^{\prime}-f_{m p} \xi R L \pi^{2}\left(1-\frac{1}{4 \eta}\right)^{2}},
$$

In this formula, the first term of the numerator takes into account the effect of bending of the shell and winding, the second - the effect of deformations of the middle surface, and the third - the effect of the winding stiffness on tension. The third term included in the denominator characterizes the influence of friction forces between the shell and the winding during their mutual displacement at the moment of buckling.

\section{Conclusions}

To obtain the value of the critical force, it is necessary to minimize the values $N_{0}(20)$ by the parameters of the shell deflection, that is, to find the dimensions of the indentations that will correspond to the minimum force. Since in formula (5) the value $f$ is expressed in terms of $\eta$, the minimization $N_{0}$ will be carried out by $\eta$, characterizing the dent width.

Minimization can be carried out by finding the derivative $\partial N_{0} / \partial \eta$ and equating it to zero, however, such an equation is transcendental, and its solution is a very difficult problem. Therefore, it seems easier to carry out minimization using numerical analysis.

The value of the minimum critical effort according to the $N_{0}$ formula (20) is determined by varying the parameter $\eta$ in the range from 1 to 60 with a step of 1 . When determining the critical force, one can vary the flexibility of the shell, its relative length, the reduced thickness of the winding, the value of the initial deflections and the friction coefficient. Taking in the formula (20) $f_{m p}=0$ we determine the value of critical efforts without taking into account the friction forces.

Summing up, we can draw the following conclusions: the given solution allows you to determine the value of critical efforts (or stresses $\sigma_{\kappa p}=N_{\kappa p} / t_{1}$ ) taking into account all the main factors [11]:

- shell length;

- shell flexibility;

- prestressing zone length;

— initial error value;

- reduced winding thickness;

- frictional forces between the shell and winding. 


\section{References}

1. N. A. Alfutov, Fundamentals of calculation for the stability of systems (1978)

2. N. A. Alfutov, On the influence of boundary conditions on the value of the upper critical pressure of a cylindrical shell (1965)

3. V. L. Berezin, V. E. Shutov, Strength and stability of tanks and pipelines (1967)

4. A. V. Karmishin, V. N. Myachenkov, A. N. Frolov, Statics and dynamics of thinwalled shell structures (1975)

5. L. G. Donell, Tanks, plates and shells (1982)

6. A. S. Volmir, Reports of the USSR Academy of Sciences, 113 (2) (1957)

7. N. A. Alumäe, Applied mathematics and mechanics, 1 (1949)

8. V. Z. Vlasov, General theory of shells and its applications to technology (1949)

9. V. Y. Vyalkov, V. A. Ivanov, V. N. Paimushin, J. of Applied Mathematics and Mechanics, 4 (2005)

10. V. V. Kozlov, Differential Equations, 4 (2009)

11. T. M. Chapaev, Scientific and Practical J. "Proceedings of Kabardino-Balkarian State Agrarian University named after V. M. Kokov", 1(15) (2017) 